21st Particles and Nuclei International Conference (PANIC 2017)

International Journal of Modern Physics: Conference Series

Vol. 46 (2018) 1860042 (6 pages)

(C) The Author(s)

DOI: $10.1142 / \mathrm{S} 201019451860042 \mathrm{X}$

\title{
More Results from the Opera Experiment at the Gran Sasso Underground Lab
}

\author{
Mustafa Kamiscioglu (on behalf of the Opera Collaboration) \\ Physics Department, Middle East Technical University \\ Ankara, TR-06800, Turkey \\ mkamisci@metu.edu.tr
}

Published 3 May 2018

\begin{abstract}
The OPERA experiment reached its main goal by proving the appearance of $\nu_{\tau}$ in the CNGS $\nu_{\mu}$ beam. Five $\nu_{\tau}$ candidates fulfilling the analysis defined in the proposal were detected with a S/B ratio of about ten allowing to reject the null hypothesis at $5.1 \sigma$. The search has been extended by loosening the selection criteria in order to obtain a statistically enhanced, lower purity, signal sample. One such interesting neutrino interaction with a double vertex topology having a high probability of being a $\nu_{\tau}$ interaction with charm production is reported. Based on the enlarged data sample the estimation of $\Delta m_{23}^{2}$ in appearance mode is presented. The search for $\nu_{e}$ interactions has been extended over the full data set with a more than twofold increase in statistics with respect to published data. The analysis of the $\nu_{\mu} \rightarrow \nu_{e}$ channel is updated and the implications of the electron neutrino sample in the framework of the $3+1$ neutrino model is discussed. An analysis of $\nu_{\mu} \rightarrow \nu_{\tau}$ interactions in the framework of the sterile neutrino model has also been performed. Finally, the results of the study of charged hadron multiplicity distributions is presented.
\end{abstract}

Keywords: Neutrino; emulsion; $\nu_{\tau}$ appearance.

\section{The OPERA experiment}

The Oscillation Project with Emulsion tRacking Apparatus(OPERA) experiment was designed to search for $\nu_{\tau}$ appearance in an almost pure $\nu_{\mu}$ beam. The OPERA detector was located in the underground Gran Sasso Laboratory (LNGS), $730 \mathrm{~km}$ away from the neutrino source, at CERN ${ }^{1-2}$. The neutrino beam, produced by $400 \mathrm{GeV}$-protons accelerated in the SPS, had an average energy of about $17 \mathrm{GeV}$, optimized for the observation of $\nu_{\tau}$ charged current (CC) interactions in the OPERA detector. In terms of interactions, the $\nu_{\mu}$ contamination was $2.1 \%$, the $\nu_{e}$ and $\overline{\nu_{e}}$ contaminations were together below $1 \%$, while the number of prompt $\nu_{\tau}$ negligible.

This is an Open Access article published by World Scientific Publishing Company. It is distributed under the terms of the Creative Commons Attribution 4.0 (CC-BY) License. Further distribution of this work is permitted, provided the original work is properly cited. 
A CC $\nu_{\tau}$ interaction in the lead-emulsion target can be identified by detecting the decay of the short-lived $\tau$ lepton in the high space-resolution nuclear emulsions.

The OPERA detector was composed of two identical super modules, each consisting of a target region followed by a muon spectrometer. The target had an overall mass of $\approx 1.25 \mathrm{kt}$ and a modular structure with approximately 150000 units, called bricks. A brick was made of $561 \mathrm{~mm}$-thick lead plates, acting as target, interleaved with 57 nuclear emulsion films, used as a micro-metric tracking device. Each film was composed of two $44 \mu \mathrm{m}$-thick emulsion layers on both sides of a $205 \mu \mathrm{m}$-thick plastic base. Bricks were arranged in walls interleaved with planes of scintillator strips forming the Target Tracker (TT). Each brick was a stand-alone device allowing momentum measurement through Multiple Coulomb Scattering (MCS) ${ }^{3}$ in the lead plates, and electromagnetic shower energy reconstruction. For each event the information provided by the electronic detectors allows identifying the brick containing the neutrino interaction, the muon charge and the momentum determination.

\section{Discovery of $\nu_{\mu} \rightarrow \nu_{\tau}$ in the CNGS neutrino beam}

During the physics runs from year 2008 to 2012, OPERA collected data corresponding to $1.8 \times 10^{20}$ protons on target. The electronic detectors recorded 19505 neutrino interactions in the target. Decay channels of observed $\nu_{\tau}$ events are given in Tab. 1 4-6. Expected background events amounted to $0.25 \pm 0.05$. Main sources of background are misidentified charmed events, hadronic re-interactions and large-angle muon scattering. Given the low background and observed candidate events, the discovery of $\nu_{\tau}$ appearance was reported with a significance of $5.1 \sigma^{6}$.

Table 1. Decay channels of observed $\nu_{\tau}$ events.

\begin{tabular}{cc}
\hline Decay Channel & Observed \\
\hline$\tau \rightarrow 1 h$ & 3 \\
$\tau \rightarrow 3 h$ & 1 \\
$\tau \rightarrow \mu$ & 1 \\
\hline
\end{tabular}

\section{3. $\Delta m_{23}^{2}$ and $\nu_{\tau}$ cross-section measurements}

In order to increase the number of $\nu_{\tau}$ candidates and to reduce the statistical error, a new search strategy was implemented based on looser kinematical cuts and multivariate analysis. Five additional $\nu_{\tau}$ candidates were collected, with a signal to background ratio reduced from 10 to 3 . The $\Delta m_{23}^{2}$ has been evaluated using the Feldman-Cousins method. Given 10 observed events with $6.8 \pm 1.4$ expected signal and $2.0 \pm 0.5$ expected background events, the result is:

$$
\Delta m_{23}^{2}=(2.7 \pm 0.6) \times 10^{-3} e V^{2} \text { at } 68 \% \text { C.L. }
$$


The $\Delta m_{23}^{2}$ value is in agreement with the PDG 2016 value $^{8}$ within $1 \sigma$. The $\nu_{\tau}$ cross-section determined assuming $\Delta m_{23}^{2}=2.5 \times 10^{-3} \mathrm{eV}^{2}$ and maximal mixing is:

$$
\sigma_{\nu_{\tau}}^{0}=\left(8_{-3}^{+4}\right) \times 10^{-39} \mathrm{~cm}^{2} \mathrm{GeV}^{-1} \text { at } 68 \% \text { C.L. }
$$

\section{4. $\nu_{\mu} \rightarrow \nu_{e}$ oscillations}

Thirty-five $\nu_{e}$ events were observed. Most of them are CC interactions of the $\nu_{e}$ and $\overline{\nu_{e}}$ beam components, other contributions are $\nu_{\tau}$ CC interactions with $\tau$ decaying into electron and $\nu_{\mu}$ event with $\pi^{0}$ misidentified as electron.

Using the values $\theta_{13}, \theta_{23}, \Delta m_{a t m}^{2}$ and the standard parameterization of the mixing matrix $\mathrm{U}$, taken from ${ }^{8}$, the total number of expected $\nu_{e}$ candidates is $34.6 \pm 3.2$. No matter effects were taken into account. The number of observed events is compatible with the 3-flavour oscillation model.

OPERA $\nu_{e}$ data sample has been used to set limits on the oscillation parameters of a massive sterile neutrino in the $3+1$ neutrino hypothesis. $\Delta m_{41}^{2}$ and $\sin ^{2}\left(2 \theta_{\mu e}\right)=$ $4\left|U_{e 4}\right|^{2}\left|U_{\mu 4}\right|^{2}$ are the parameters of interest. Preliminary $90 \%$ C.L. exclusion plot obtained by OPERA experiment is shown in Fig. 1.

\section{Event with two secondary vertices}

OPERA detected a neutral current like interaction with two secondary vertices. The total hadronic energy is about $20 \mathrm{GeV}$. The primary vertex $\left(V_{I}\right)$, in Fig. 2, is 581.8 $\mu m$ upstream of the top emulsion layer of plate 32 , while the secondary vertex $\left(V_{I I}\right)$ is just $102.6 \mu \mathrm{m}$ downstream of $V_{I}$. The primary vertex is composed by tracks 2,4 and 5 , while $V_{I I}$ is composed by tracks 1 and 3 . Track 4 (parent) exhibits a kink with track 6 (daughter). The kink point is labelled as $V_{I I I}$.

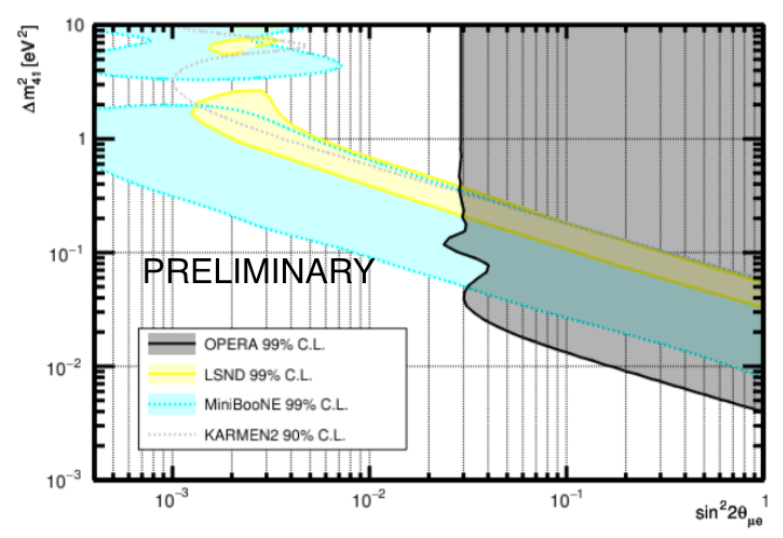

Fig. 1. Preliminary $90 \%$ C.L. exclusion plot in the $\Delta m_{41}^{2}$ and $\sin ^{2}\left(2 \theta_{\mu e}\right)$ plane. Allowed regions obtained by LSND, MiniBooNE and Karmen experiments are also shown. 


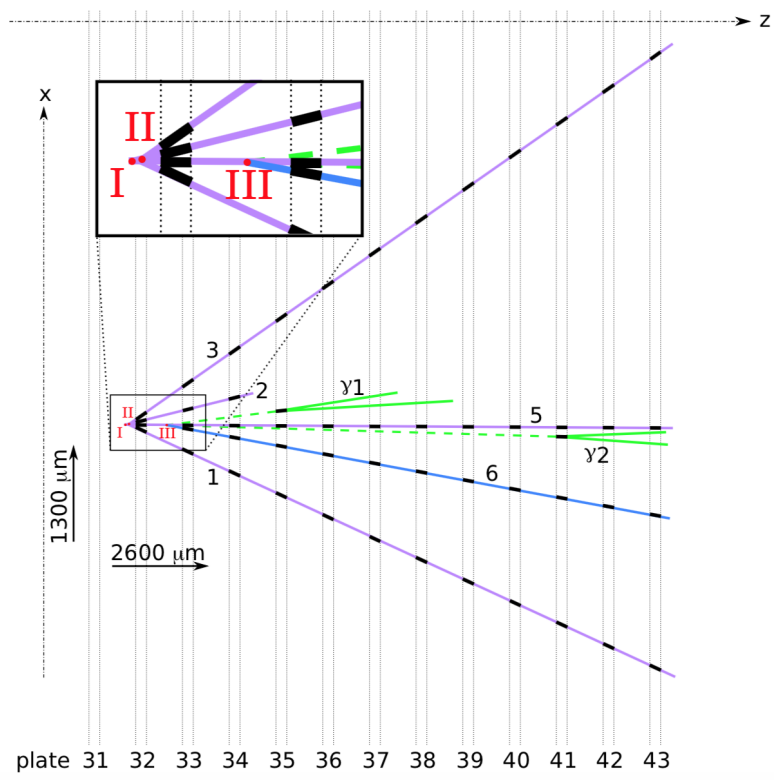

Fig. 2. Projection of the event with two secondary vertices in xz plane. The parent (track 4) is not visible since it is behind track 5 . For photons, only the first electron-positron pair is shown.

Dedicated simulations and Artificial Neural Networks analysis was performed to distinguish between possible interpretations. The most likely one is the vertex II is originated by a charm decay and vertex III by a tau decaying into a hadron. The event is classified as a $\nu_{\tau} \mathrm{CC}$ interaction with charm production with a significance of $3.5 \sigma$.

\section{Study of charged hadron multiplicity distributions}

The multiplicity distribution of charged hadrons is an important characteristic since it reflects the dynamics of the interaction. An unbiased sub-sample of $\nu_{\mu} \mathrm{CC}$ interactions occurring in the lead was selected. Only events with the square of the invariant mass of the hadronic system $\left(W^{2}\right)$ larger than $1 \mathrm{GeV}^{2} / \mathrm{c}^{4}$ were used in order to eliminate the quasi-elastic contribution.

Selected $\nu_{\mu}$ CC events were inspected carefully and tracks were classified depending on their ionization features: minimum ionization particle (mip), grey and black. The mip tracks are highly relativistic charged particles generated by neutrinonucleon interaction. Black tracks are produced by low energy fragments. Grey tracks are produced by slow particles interpreted as recoil nucleons emitted during the nuclear cascade. The charged hadron multiplicity $\left(\left\langle n_{c h}\right\rangle\right)$ is defined as the number of mip tracks $\left(\left\langle n_{m i p}\right\rangle\right)$ excluding the muon track, so $\left\langle n_{c h}\right\rangle=\left\langle n_{m i p}\right\rangle-1$. Multiplicity distribution of mip tracks is shown in Fig 3.

The average charged hadron multiplicity $\left(\left\langle n_{c h}\right\rangle\right)$ is well described by a linear function in $\ln W^{2}$ shown in Fig. 4 (left). The dispersion $\left(D_{c h}\right)$ is defined as $D_{c h}=$ 


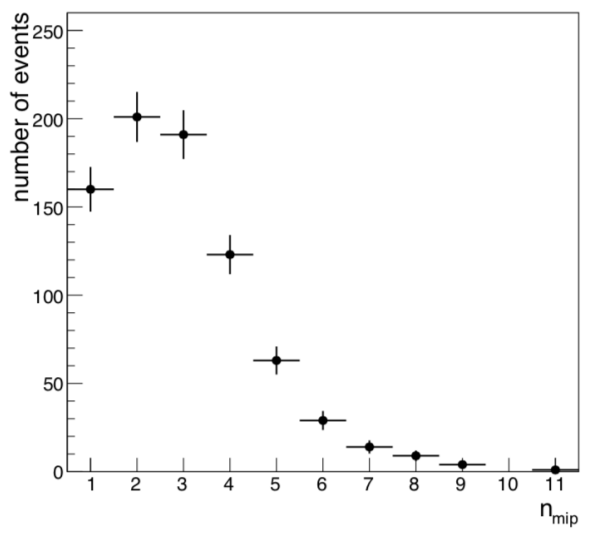

Fig. 3. Multiplicity distribution of mip tracks.
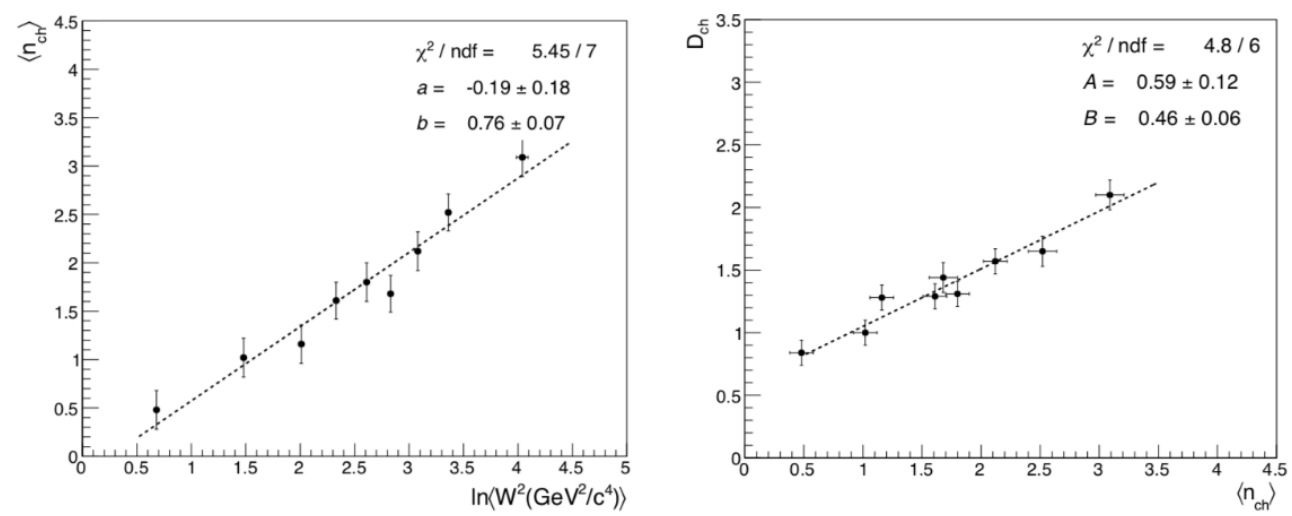

Fig. 4. (Left panel) average charged hadron multiplicity distributions as a function of $\ln W^{2}$. (Right panel) Average charged hadron multiplicity dispersion as a function of $\left\langle n_{c h}\right\rangle$.

$\sqrt{\left\langle n_{c h}{ }^{2}\right\rangle-\left\langle n_{c h}\right\rangle^{2}}$. The dispersion as a function of $\left\langle n_{c h}\right\rangle$ is presented in Fig. 4 (right). The dependence is approximately linear.

\section{Conclusions}

After the discovery of the $\nu_{\tau}$ appearance in the CNGS neutrino beam, an extended analysis has been performed to increase number of $\nu_{\tau}$ candidate events. Based on the enlarged data sample the values of $\Delta m_{23}^{2}$ in appearance mode and the $\nu_{\tau}$ crosssection determined.

The $\nu_{\mu} \rightarrow \nu_{e}$ oscillation search results are updated: the number of observed events is in agreement with the expected background in the 3-flavour oscillation model. An upper limit to the mixing with a fourth sterile neutrino is set. 
Moreover OPERA detected an event classified as a $\nu_{\tau} \mathrm{CC}$ interaction with charm production. The study of the multiplicity distribution of charged hadron particles in neutrino-lead interactions is presented.

\section{References}

1. OPERA Collab. (R. Acquafredda et al.), JINST 4, P04018 (2009).

2. OPERA Collab. (R. Acquafredda et al.), New J. Phys. 8, 303 (2006).

3. OPERA Collab. (N.Agafonova et al.), New J. Phys. 14, 013026 (2012).

4. OPERA Collab. (N.Agafonova et al.), Phys. Lett. B 691, 138 (2010).

5. OPERA Collab. (N.Agafonova et al.), Prog. Theor. Exp. Phys., 101C01 (2014).

6. OPERA Collab. (N.Agafonova et al.), Phys. Rev. Lett 115, 121802 (2015).

7. OPERA Collab. (N.Agafonova et al.), JHEP 04, 014 (2014).

8. PDG Collab. (C. Patrignani et al.), Review of Particle Physics. Chin. Phys. C40(10), 100001 (2016).

9. OPERA Collab. (N.Agafonova et al.), Phys. Rev. D 89, 051102 (2014). 